九州大学学術情報リポジトリ

Kyushu University Institutional Repository

\title{
ON EXPANSION OF ESTIMATES FOR MEAN VALUE FUNCTIONS OF HOMOGENEOUS RANDOM FIELDS ON COMPACT HOMOGENEOUS SPACES
}

Nagai, Takeaki

Faculty of Engineering Science, Osaka University

https://doi.org/10.5109/13061

出版情報: 統計数理研究. 15 (1/2)，pp. 39-42，1972-03. Research Association of Statistical Sciences

バージョン：

権利関係 : 


\title{
ON EXPANSION OF ESTIMATES FOR MEAN VALUE FUNCTIONS OF HOMOGENEOUS RANDOM FIELDS ON COMPACT HOMOGENEOUS SPACES
}

\author{
By \\ Takeaki NAGAI* \\ (Received November 5, 1971)
}

\section{§1. Summary.}

We consider the uniformly minimum variance unbiased linear estimators $\hat{m}(t)$ of mean value functions $m(t)$ of homogeneous random fields on compact homogeneous spaces and their expansions by spherical functions.

\section{§2. Expansions of estimates for mean value functions.}

Let $T=\{t\}$ be an arbitrary compact homogeneous space, that is, a compact space which admits a transitive transformation group $G=\{g\}$. We denote by $K=\{k\}$ a stationary subgroup of $G$, that is, a subgroup which leaves invariant a point $t_{0} \in T$.

Let $\{X(t), t \in T\}$ be a real-valued homogeneous random field on $T$ having the mean value function

$$
m(t)=E\{X(t)\}, \quad t \in T
$$

and satisfying the conditions :

$$
E\left\{|X(t)|^{2}\right\}<\infty, \quad \text { for all } t \in T .
$$

(C.2) The covariance functions

$$
R(t, s)=E\{(X(t)-m(t))(X(s)-m(s))\}
$$

is a continuous positive definite function on $T \times T$.

(C.3) For all $g \in G$,

$$
R(t, s)=R(g t, g s), \quad t, s \in T .
$$

We denote by $L_{2}(X)$ a Hilbert space consisting of all random variables which may be represented either as a finite linear combinations $U=\sum_{j=1}^{n} c_{j} X\left(t_{j}\right)$, for some integer $n$, points $t_{1}, t_{2}, \cdots, t_{n}$ in $T$ and scalars $c_{1}, c_{2}, \cdots, c_{n}$, or as a limit in quadratic mean of such finite linear combinations under the scalar product defined by $(U, W)$ $=E\{U \cdot W\}$.

We denote by $H(R)$ a reproducing kernel Hilbert space generated by the kernel

* Faculty of Engineering Science, Osaka University, Toyonaka, Osaka. 
$R(s, t), s, t \in T . H(R)$ is actually a Hilbert space consisting of functions on $T$ satisfying the conditions:

(R.1) For each $t \in T, R(t, \cdot) \in H(R)$.

(R.2) For any $f \in H(R)$,

$$
(f, R(t, \cdot))_{R}=f(t), \quad t \in T,
$$

where by $(\cdot, \cdot)_{R}$ we denote the scalar product in $H(R)$.

Suppose that the mean value function $m(\cdot)$ is known to be in a closed subspace $\Sigma$ of $H(R)$ but the actual values of $m(t), t \in T$, are not known.

The problem to find "the uniformly minimum variance unbiased linear estimator $\hat{m}(t) \in L_{2}(X)$ for $m(t)$ at $t$ " in the sense that for each $t \in T$ the followings hold:

$$
E_{m}\{\hat{m}(t)\}=m(t) \quad \text { for all } m \in \Sigma,
$$

(E.2) For any unbiased estimate $\hat{\hat{m}}(t)$,

$$
\operatorname{Var}(\hat{m}(t)) \leqq \operatorname{Var}(\hat{\hat{m}}(t)), \quad \text { uniformly in } m \in \Sigma \text {. }
$$

is completely solved (E. Parzen [2]), where by $E_{m}\{\cdot\}$ we denote the expectation is calculated by the probability with its mean value function $m(\cdot)$.

This solution $\hat{m}(t)$ is given by

$$
\hat{m}(t)=\phi(\operatorname{Proj}(R(t, \cdot) \mid \Sigma)), \quad t \in T
$$

where $\phi$ is a congruence between $H(R)$ and $L_{2}(X)$, (E. Parzen [2], [3]), such that, for $f, g \in H(R)$,

$$
\begin{gathered}
\phi(R(t, \cdot))=X(t), \quad t \in T, \\
E_{m}\{\phi(f)\}=(f, m)_{R}, \quad \text { for all } m \in \Sigma, \\
\operatorname{Cov}(\psi(f), \phi(g))=(f, g)_{R} .
\end{gathered}
$$

We consider here the case where the subspace $\Sigma$ is invariant under $K$, that is, $\Sigma=\{f \in H(R) \mid f(t)=f(k t), k \in K\}$.

In this case, we shall see that the estimator $\hat{m}(t)$ can be expressed quite in a simple form by making use of zonal spherical functions.

Let $\left\{T^{(\lambda)}(g)=\left[T_{i j}^{(\lambda)}(g)\right], g \in G, \lambda=1,2, \cdots, 1 \leqq i, j \leqq d_{\lambda}\right\}$ be the complete system of unitary continuous non-equivalent representations of the group $G$ and let us choose in the space of these representations a basis such that these representations decompose into irreducible representations of the subgroup $K$. Suppose, for instance, that the representation $T^{(\lambda)}$ of the group $G$ contains $r_{\lambda}$ times the identity representation of $K$. Suppose that in our basis $l_{1}, l_{2}, \cdots, l_{d_{\lambda}}$, these identity representations correspond to the first $r_{\lambda}$ basis vectors so that $T^{(\lambda)}(k) l_{j}=l_{j}$ for $k \in K$ and $j=1,2, \cdots, r_{\lambda}$.

In this case the functions of $t$,

$$
\Phi_{i j}^{(\lambda)}(t)=T_{i j}^{(\lambda)}(g) ; \quad i=1,2, \cdots, d_{\lambda}, j=1,2, \cdots, r_{\lambda}, \lambda=1,2,3, \cdots,
$$

will be called spherical functions over $T$, while the functions

$$
\Phi_{i j}^{(\lambda)}(t)=T_{i j}^{(\lambda)}(g) ; \quad i, j=1,2, \cdots, r_{\lambda}, \lambda=1,2,3, \cdots
$$


will be called zonal spherical functions over $T$. (The matrix elements $T_{i j}^{(\lambda)}(g)$ are constant over all left cosets of $G$ modulo $K$.)

Under these notations, we have the following theorem:

THEOREM : Let the mean function $m(\cdot)$ be invariant under the subgroup $K$ of $G$, that is,

$$
\Sigma=\{f \in H(R) \mid f(t)=f(k t), k \in K\} .
$$

Then the uniformly minimum variance unbiased linear estimator $\hat{m}(t)$ for $m(t)$ at $t$ has the following expansion:

where

$$
\hat{m}(t)=\sum_{\lambda=1}^{\infty} \sum_{i=1}^{r_{\lambda}} \sum_{j=1}^{r_{\lambda}} X_{i j}^{(\lambda)} \cdot Q_{i j}^{(\lambda)}(t), \quad t \in T
$$

$$
\begin{gathered}
Q_{i j}^{(\lambda)}(t)=\Phi_{i j}^{(\lambda)}(t) /\left\|\Phi_{i j}^{(\lambda)}\right\| \\
X_{i j}^{(\lambda)}=\int_{T} X(s) Q_{i j}^{(\lambda)}(s) d s \\
\left\|\Phi_{i j}^{(\lambda)}\right\|^{2}=\int_{T}\left|\Phi_{i j}^{(\lambda)}(s)\right|^{2} d s .
\end{gathered}
$$

Proof. Let $r(\hat{g})=R\left(\hat{g} t_{0}, t_{0}\right)$. Then, since for any $g \in G R(t, s)=R(g t, g s)$, we see that for each $k \in K r(\hat{g})=r\left(k^{-1} \hat{g} k\right)$, that is, $r(\cdot)$ is a class function of $K$. Hence, from the Yaglom's result (Yoglem [4], Theorem 5, p. 604) the covariance function $R(t, s)$ can be represented in the form;

Hence, we have

$$
R(t, s)=\sum_{\lambda=1}^{\infty} \sum_{i=1}^{r_{\lambda}} \sum_{j=1}^{r_{\lambda}} f_{i j}^{(\lambda)} \cdot \sum_{i=1}^{d_{\lambda}} \Phi_{i i}^{(\lambda)}(t) \Phi_{i j}^{(\lambda)}(s)
$$

$$
\begin{aligned}
R_{1}\left(t_{1}, \cdot\right) & =\operatorname{Proj}(R(t, \cdot) \mid \Sigma) \\
& =\sum_{\lambda=1}^{\infty} \sum_{i=1}^{r_{\lambda}} \sum_{j=1}^{r_{\lambda}} f_{i j}^{(\lambda)} \sum_{i=1}^{r_{\lambda}} \Phi_{i i}^{(\lambda)}(t) \cdot \Phi_{i j}^{(\lambda)}(\cdot) .
\end{aligned}
$$

Since $\int_{T} \int_{T} R(t, s) \Phi_{i j}^{(\lambda)}(t) \Phi_{i j}^{(\lambda)}(s) d t d s=f_{i j}^{(\lambda)} \cdot\left\|\Phi_{i j}^{(\lambda)}\right\|^{4}<\infty$,

$$
U_{i j}^{(\lambda)}=\int_{T} X(s) \Phi_{i j}^{(\lambda)}(s) d s
$$

is well-defined and therefore there exists a unique function $p_{i j}^{(\lambda)} \in H(R)$ such that $\phi\left(p_{i j}^{(\lambda)}\right)=U_{i j}^{(\lambda)}$.

The function $p_{i j}^{(i)}$ can be easily determined and given by

$$
p_{i j}^{(\lambda)}(t)=\left\|\Phi_{i j}^{(\lambda)}\right\|^{2} \cdot \sum_{i=1}^{r} f_{j i}^{(\lambda)} \Phi_{i l}^{(\lambda)}(t)
$$

Thus, we have

$$
U_{i j}^{(\lambda)}=\left\|\Phi_{i j}^{(\lambda)}\right\|^{2} \cdot \phi\left(\sum_{l=1}^{r_{\lambda}} f_{j l}^{(\lambda)} \cdot \Phi_{i l}^{(\lambda)}(\cdot)\right) .
$$

Let us write

$$
X_{i j}^{(\lambda)}=U_{i j}^{(\lambda)} /\left\|\Phi_{i j}^{(\lambda)}\right\|=\int_{T} X(s) Q_{i j}^{(\lambda)}(s) d s
$$

where 


$$
Q_{i j}^{(\lambda)}(t)=\Phi_{i j}^{(\lambda)}(t) /\left\|\Phi_{i j}^{(\lambda)}\right\| .
$$

Then, we have the following expansion

$$
\begin{aligned}
\hat{m}(t)=\psi\left(R_{1}(t, \cdot)\right) & =\sum_{i=1}^{\infty} \sum_{i=1}^{r_{\lambda}} \sum_{j=1}^{r_{\lambda}} \Phi_{i i}^{(\lambda)}(t) \phi\left(\sum_{j=1}^{r_{\lambda}} f_{i j}^{(\lambda)} \Phi_{i j}^{(\lambda)}(\cdot)\right) \\
& =\sum_{\lambda=1}^{\infty} \sum_{i=1}^{r_{\lambda}} \sum_{i=1}^{r_{\lambda}} Q_{i i}^{(\lambda)}(t) \cdot X_{i i}^{(\lambda)} .
\end{aligned}
$$

Example (NAgAi [1]). Let $T=\left\{(x, y, z) \mid x^{2}+y^{2}+z^{2}=1\right\}$ and $x=\sin \theta \cos \phi, y=$ $\sin \theta \sin \psi$ and $z=\cos \theta ; 0 \leqq \theta \leqq \pi, 0 \leqq \psi<2 \pi$. Let $\{X(t), t \in T\}$ be a homogeneous random field on $T$ and its mean value function $m(\theta, \phi)$ be invariant under the rotation group $K=S O(2)$. Then the uniformly minimum variance unbiased linear estimator $\hat{m}(\theta, \phi)$ at $(\theta, \phi) \in T$ can be expressed as follows;

$$
\hat{m}(\theta, \phi)=\sum_{v=0}^{\infty} X_{v} \cdot \phi_{v}(\theta), \quad 0 \leqq \theta \leqq \pi, 0 \leqq \phi \leqq 2 \pi,
$$

where

$$
\begin{gathered}
\phi_{v}(\theta)=\sqrt{2 v+1} P_{v}(\cos \theta), \\
X_{v}=\frac{1}{4 \pi} \int_{0}^{\pi} \int_{0}^{2 \pi} X(\theta, \phi) \phi_{v}(\theta) \sin \theta d \phi d \theta
\end{gathered}
$$

and

$$
\left\{P_{v}(z), \quad|z| \leqq 1, \quad v=0,1,2, \cdots\right\}
$$

are Legendre's polynomials.

\section{References}

[1] NaGaI, T.; On regression analysis of a certain random field on the unit sphere. Research Report of Kagoshima Univ. No. 1, (1968), 25-32.

[2] PARzen, E.; A new approach to the synthesis of optimal smoothing and prediction systems. Tech. Report, (1960), No. 34, Stanford Univ.

[3] Parzen, E.; An Approach to time series analysis. Ann. Math. Statist., 32 (1961), 951989.

[4] YaGlom, A.M.; Second order homogeneous random fields. Proc. Fourth Berkeley Symp. on Math. Stat. and Prob. 2, (1961), 593-622. 